\title{
Comparison of Back and Top Gating Schemes with Tunable Graphene Fractal Metasurfaces
}

\author{
Ayse Melis Aygar, ${ }^{*}, \dagger$, Osman Balci, ${ }^{\S}$ Semih Cakmakyapan, ${ }^{\dagger, \perp}$ Coskun Kocabas, ${ }^{\S}$ Humeyra Caglayan, ${ }^{\dagger}$ \\ and Ekmel Ozbay ${ }^{\dagger}, \S$
}

\author{
${ }^{\dagger}$ Nanotechnology Research Center, ${ }^{\ddagger}$ Department of Electrical and Electronics Engineering, and ${ }^{\S}$ Department of Physics, Bilkent \\ University, Bilkent, Ankara 06800, Turkey \\ ${ }^{\perp}$ Electrical Engineering Department, University of California Los Angeles, Los Angeles, California 90095, United States
}

\section{Supporting Information}

ABSTRACT: In this work, fractal metasurfaces that consist of periodic gold squares on graphene are used to increase lightgraphene interaction. We show by simulations and experiments that higher level fractal structures result in higher spectral tunability of resonance wavelength. This is explained by higher field localization for higher level fractal structures. Furthermore, spectral tunability of fractal metasurfaces integrated with graphene is investigated comparing two different schemes for electrostatic gating. Experiment results show that a top-gated device yields more spectral tunability ( $8 \%$ of resonance wavelength) while requiring much smaller gate voltages
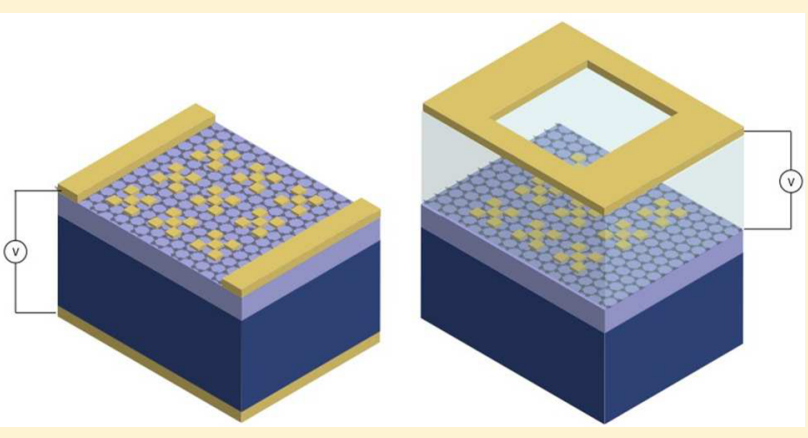
compared to the back-gated device.

KEYWORDS: graphene, plasmonics, spectral tunability, fractal metasurfaces

raphene is a $2 \mathrm{D}$ material that consists of carbon atoms

arranged in a honeycomb lattice. It has been a promising material for electro-optic devices due to various remarkable properties. Graphene has a very high carrier mobility ${ }^{1}$ owing to zero effective mass of traveling electrons ${ }^{2}$ and electron mean free path values on the order of micrometers. ${ }^{3}$ Furthermore, optical transitions in graphene can be largely controlled by electrical gating due to confinement of electrons in one atomic layer and to a remarkable shift of the Fermi level with varying carrier concentration. ${ }^{4}$ Thus, graphene is a good choice of material for field-effect devices. Carrier concentration dependent optical conductivity of graphene also allows for the tunability of plasmon resonances of graphene-hybrid devices. Tuning ranges of $8 \%{ }^{5}$ and $20 \%{ }^{6}$ of the plasmon resonance frequency were reported by Yao et al. previously.

Traditional back gating of graphene on a highly doped $\mathrm{Si}$ / $\mathrm{SiO}_{2}$ substrate is a reliable method to manipulate its carrier concentration. In this approach, control voltages increase as the oxide layers become thicker, and control voltages as high as 390 $\mathrm{V}$ may be necessary for a device with a $1 \mu \mathrm{m}$ thick oxide layer. ${ }^{7}$ Back-gated graphene devices have been used in many applications as photodetectors, ${ }^{8}$ modulators, ${ }^{7,9}$ and nanoresonators. ${ }^{10}$ On the other hand, a more efficient dielectric, an ion gel, has been used by Halas et al. ${ }^{11}$ and Ju et al. ${ }^{12}$ to top gate a patterned graphene layer with much lower control voltages.

In this work, we investigated graphene-gold fractal metasurfaces to enhance light-graphene interaction. The system consists of periodic gold fractal squares with three different levels patterned on a $\mathrm{Si}-\mathrm{SiO}_{2}$-graphene substrate. Furthermore, measurements are made using two separate devices with different gating schemes, and the results are compared. Carrier density of graphene is manipulated by using back gating and $\mathrm{SiO}_{2}$ dielectric for the first device. The second device is top gated using ionic liquid as a conducting medium. In both devices, the tunability of the resonances is promising for filtering and switching applications.

\section{DESIGN AND EXPERIMENTS}

The layouts of the two devices are shown in Figure 1. In both devices, $\mathrm{Si} / \mathrm{SiO}_{2} /$ graphene substrates are used. On these substrates, there are different metasurfaces, which consist of gold periodic fractals of different levels. In the first device (Figure 1a), source and drain contacts on graphene and a back contact enable gating graphene. In the second device (Figure 1b) graphene is gated through a top contact and an ionic liquid electrolyte that lies in between graphene and the top contact.

Fractal metasurfaces serve the purpose of increasing graphene-light interaction. Fractal level is defined as the number of self-repetitions of the geometry. First-, second-, and third-level square fractal patterns are designed in order to compare the effects of plasmonic interaction. The periodicity, $p$, and the side length of the smallest square unit, $a$, is chosen to achieve resonance peaks at similar wavelengths (around 6-6.5

Received: June 26, 2016

Published: November 1, 2016 


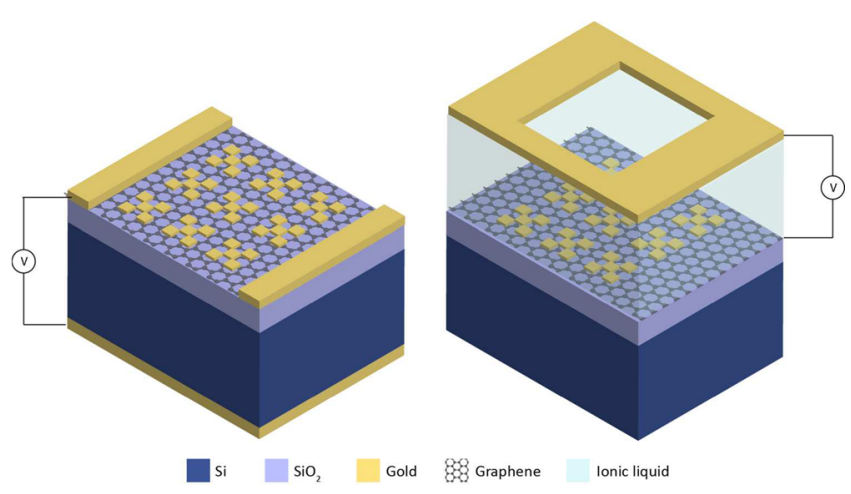

Figure 1. Isometric views of the measured devices that consist of gold fractal structures fabricated on graphene on a p-doped $\mathrm{Si}$ substrate with a $280 \mathrm{~nm}$ thick $\mathrm{SiO}_{2}$ layer. Back gate (a) and top gate (b) geometries are utilized.

$\mu \mathrm{m})$. For first-, second-, and third-level fractals $(p, a)$ values are $(2400,640),(1800,150)$, and $(1600,40) \mathrm{nm}$, respectively (see Figure S1 in the Supporting Information). To compare the electric field localizations for different fractal patterns, a set of simulations without graphene is carried out. Figure $2 d-f$ show the electric field distribution at the resonance wavelengths taken from a monitor that lies on the $\mathrm{SiO}_{2}$ /gold interface where graphene would lie. The mesh size used is $5 \mathrm{~nm}$ in the $x$ and $y$ directions and $0.25 \mathrm{~nm}$ in the $z$ direction. The electric field distribution shows that the intensity of the localized field and the number of localization centers both increase going from first- to third-level fractals. The effective mode area of each fractal level is calculated as $2.71,2.06$, and $1.83 \mu \mathrm{m}^{2}$ for first-, second-, and third-level fractals, respectively. The EM density, which is the ratio of the effective mode area of each unit cell to the total area of the unit cell, is calculated as $0.47,0.64$, and 0.715 going from first- to third-level fractals. Hence, the lightgraphene interaction is greater for higher level fractal geometries.

The rest of the simulations are carried out with graphene. In these simulations, the sheet optical conductivity of graphene is modeled as given in eq $1,{ }^{13,14}$ where $k_{\mathrm{B}}$ is the Boltzmann constant, $T$ is the temperature, $\omega$ is the frequency, $E_{\mathrm{F}}$ is the Fermi energy, and $\tau$ is the carrier relaxation lifetime. Carrier relaxation lifetime, $\tau$, depends on $\sigma$, the semiclassical diffusive conductivity for 2D graphene, $g_{s}$ and $g_{v}$, which are the spin and valley degeneracy factors, respectively, and are taken as $2 .{ }^{15}$ This dependence is given in eq 2 .

$$
\begin{aligned}
\sigma_{\mathrm{s}}(\omega)= & \frac{2 i e^{2} k_{\mathrm{B}} T}{\pi \hbar^{2} \omega+i / \tau} \ln \left[2 \cosh \left(\frac{E_{\mathrm{F}}}{2 k_{\mathrm{B}} T}\right)\right] \\
& +\frac{e^{2}}{4 \hbar}\left[\frac{1}{2}+\frac{1}{\pi} \tan ^{-1}\left(\frac{\hbar \omega-2 E_{\mathrm{F}}}{2 k_{\mathrm{B}} T}\right)\right. \\
& \left.-\frac{i}{2 \pi} \ln \left(\frac{\left(\hbar \omega+2 E_{\mathrm{F}}\right)^{2}}{\left(\hbar \omega-2 E_{\mathrm{F}}\right)^{2}+4\left(k_{\mathrm{B}} T\right)^{2}}\right)\right] \\
\tau=\sigma & \frac{\hbar}{g_{\mathrm{s}} g_{\mathrm{v}} e^{2}} \frac{2 \hbar}{E_{\mathrm{F}}}
\end{aligned}
$$

The permittivity of bulk graphene is mapped to the sheet conductivity using eq 3 , where $\varepsilon$ is the volume permittivity, $\sigma_{\mathrm{s}}$ is the sheet conductivity, and $t_{\mathrm{G}}$ is the graphene thickness. The permittivity data are later imported to the simulation software to model a $1 \mathrm{~nm}$ thick graphene material for different Fermi energy values. The thickness of our graphene material is defined to be larger than the actual thickness of single-layer graphene in order to use coarser meshes in the simulation and save simulation time.

$$
\varepsilon(\omega)=\varepsilon_{\mathrm{r}}+i \varepsilon_{i}=1+\frac{i \sigma_{\mathrm{s}}}{\omega \varepsilon_{0} t_{\mathrm{G}}}
$$

Two devices are fabricated with three different regions of fractal metasurfaces on each one. The details of the fabrication are found in the Methods section. The scanning electron microscope (SEM) images of fractal patterns on graphene are given in Figure $2 a-c$. After fabricating both devices a series of experiments were done. During the experiments, measurements
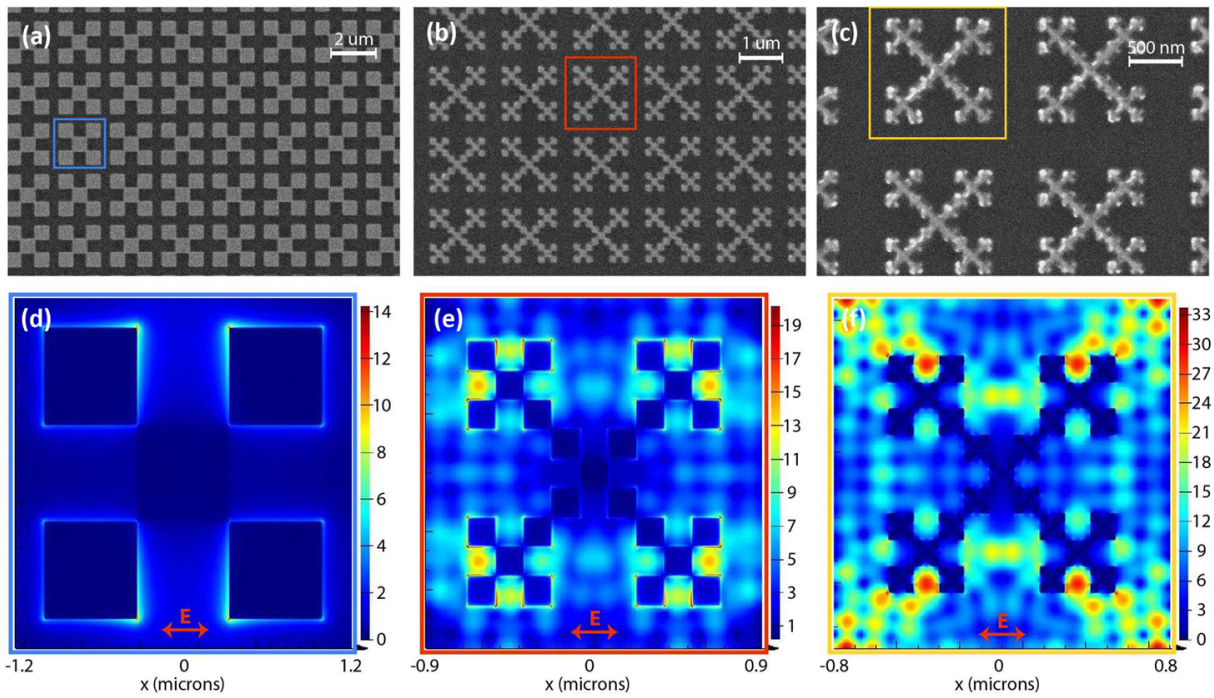

Figure 2. $(\mathrm{a}-\mathrm{c}) \mathrm{SEM}$ images of the different level periodic gold fractal structures on a $\mathrm{Si} / \mathrm{SiO}_{2} /$ graphene substrate. Unit cells are marked with colored squares. (a), (b), and (c) illustrate first-, second-, and third-level fractals, respectively. (d-f) Simulation results of the E-field intensity distribution on the $\mathrm{SiO}_{2}$ /gold pattern interface at the resonance wavelength. (d), (e), and (f) illustrate first-, second-, and third-level fractals' E-field localizations, respectively. The unit cells are symmetric in the $x$ and $y$ directions. 

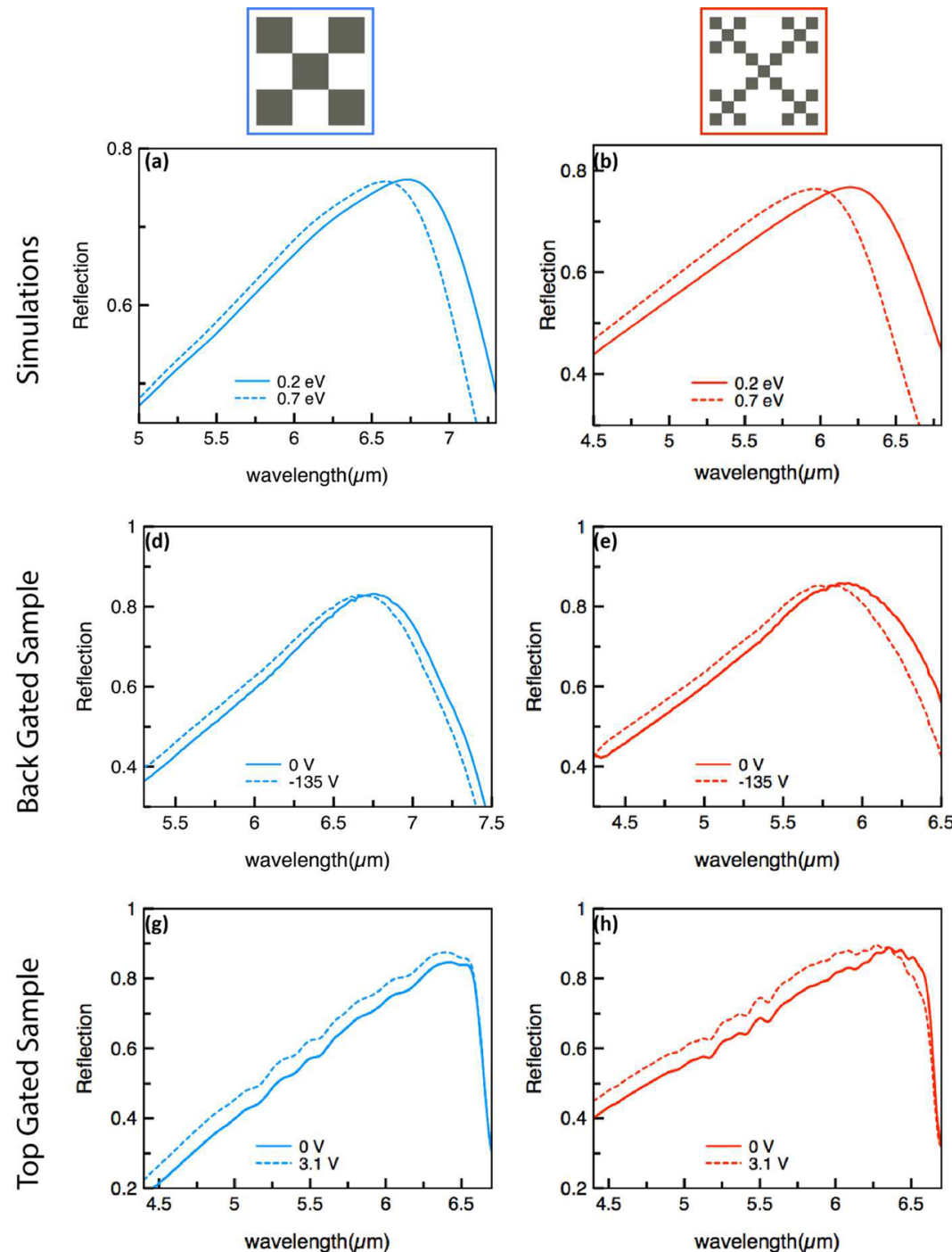
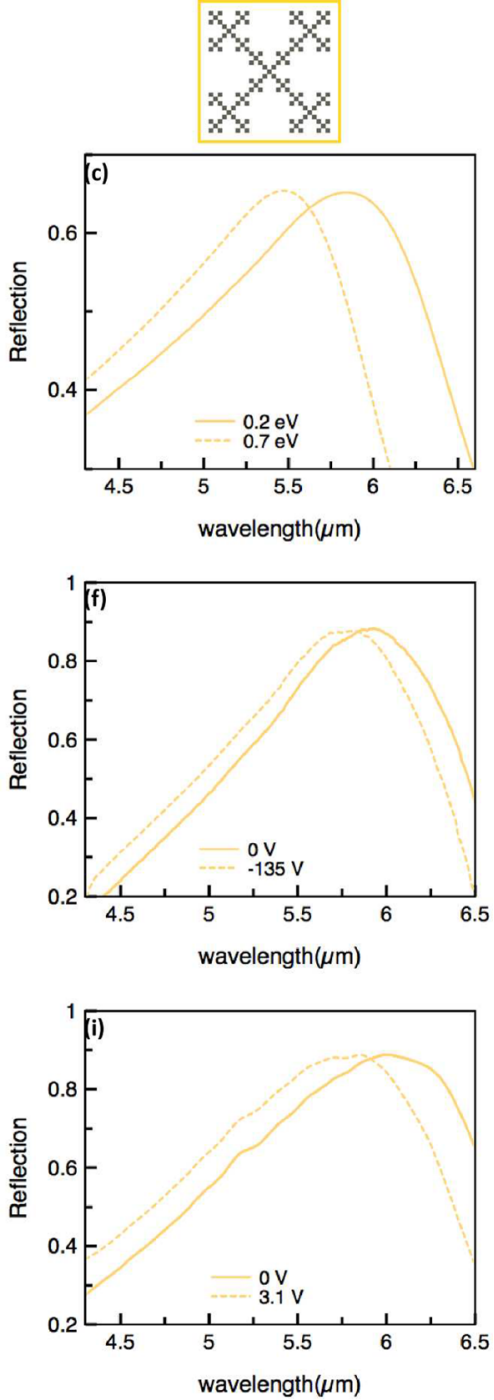

Figure 3. Normalized reflection spectra of numerical simulations, measurements with top gating, and measurements with back gating are illustrated in $(a-c),(d-f)$, and $(g-i)$ respectively. For each case levels of the fractal metasurfaces are color coded, where blue, red, and yellow correspond to first-, second-, and third-level fractals, respectively.

are taken using an FTIR spectroscopy system integrated with a microscope. Using the microscope incoming light is focused on the regions of the different fractal patterns. Reflection spectra are measured for different values of the gate voltage. In the first device (Figure $1 \mathrm{a})$, a small voltage $(0.2 \mathrm{~V})$ is applied between source and drain contacts and the gate voltage is applied between the source and back contact. In the second device (Figure 1b) gate voltage is applied between graphene and the top contact

\section{RESULTS AND DISCUSSION}

The normalized reflection spectra of numerical simulations and measurements for the three different fractal designs are illustrated in Figure 3. Figure $3 a-c$ present the results of the numerical simulations. Measurement results of back- and topgated samples are given in Figure $3 \mathrm{~d}-\mathrm{f}$ and $\mathrm{g}-\mathrm{i}$, respectively. For the back-gated sample, the charge neutrality point (CNP) is calculated using the $C=n e / V^{16}$ relationship, where $n$ is the sheet carrier concentration and $C$ is the capacitance per unit area. Capacitance per unit area is estimated as $C=1.2 \times 10^{-4}$ $\mathrm{F} / \mathrm{m}^{2}$, and Hall measurements of the graphene samples give the sheet carrier concentration at no applied voltage as $n=6.4 \times$ $10^{12} \mathrm{~cm}^{-2}$. Therefore, the CNP is expected to be at $85 \mathrm{~V}$ (see Figure S2 in the Supporting Information), and the gate voltage applied during the FTIR (Fourier transform infrared) measurements spans a voltage range from $85 \mathrm{~V}$ down to $-50 \mathrm{~V} . \Delta V$ values, which are applied gate voltages with respect to $V_{\mathrm{CNP}}$, are indicated in the legend of Figure $3 \mathrm{~d}-\mathrm{f}$ for clarity. For the topgated sample, the CNP is found at $-0.6 \mathrm{~V}$, with $I-V$ measurements plotted in Figure S3 in the Supporting Information. Gate voltages higher than $2.5 \mathrm{~V}$ induce irreversible structural deformation on graphene. ${ }^{17}$ Hence, for this sample, gate voltage applied during the FTIR measurements spans a voltage range from -0.6 to $2.5 \mathrm{~V}$. Again, $\Delta V$ values, which are applied gate voltages with respect to $V_{\mathrm{CNP}}$, are indicated in the legend of Figure $3 \mathrm{~g}-\mathrm{i}$ for clarity.

The Fermi level of graphene depends on sheet carrier concentration according to $E_{\mathrm{F}}=\hbar v_{F} \sqrt{\pi n}$. ${ }^{2}$ This relationship enables manipulation of the Fermi level by gating graphene. Fermi energy dependence of graphene permittivity according to eq 3 allows for spectral tuning of the resonance wavelength by gating graphene. Therefore, in all simulations and measure- 
ments, a resonance shift to shorter wavelengths is observed as the carrier concentration of graphene increases. For the backgated sample a voltage span of $135 \mathrm{~V}$ was required to achieve spectral tunability values of 88,162 , and $211 \mathrm{~nm}$ for first-, second-, and third-level fractal metasurfaces, respectively. For the top-gated sample, however, a voltage span of $3.1 \mathrm{~V}$ was enough to record resonance wavelength shifts of 111,207, and $378 \mathrm{~nm}$ for first-, second-, and third-level fractal metasurfaces, respectively. The top-gated sample therefore is said to be a more efficient gating scheme. The simulation and measurement results also show that the amount of wavelength shift is greater for higher level fractal structures. This relationship is due to the greater light-graphene interaction for metasurfaces with higher level fractal patterns as depicted in Figure $2 \mathrm{~d}-\mathrm{f}$. For third level fractals, the $E$-field is more localized on the graphene due to the plasmonic modes; hence more tunability of the resonance is achieved.

As a conclusion, top gating graphene using the ionic liquid electrolyte is a more efficient way for spectral tuning of resonances than the traditional back gating method. Resonance wavelength shifts as high as $378 \mathrm{~nm}$ (6\% of the resonance wavelength) are recorded by applying much less controlled voltages in the top gating method. Furthermore, it is shown that for higher level fractal metasurfaces more spectral tunability is achieved due to more light-graphene interaction. When fractal metasurfaces that localize more light are integrated with graphene, graphene's properties such as tunability are benefited more. This is very promising for the realization of graphene-based electro-optic devices.

\section{METHODS}

Simulation. The numerical simulations are carried out by using the finite-difference time domain simulation software, Lumerical FDTD Solutions. In the simulation setup, $280 \mathrm{~nm}$ thick $\mathrm{SiO}_{2}$ is layered on an infinite silicon slab. On top of that $50 \mathrm{~nm}$ thick gold squares are placed to form different level fractal unit cells. Periodic boundary conditions are used on the sides of the unit cell, while using perfect matched layer (PML) boundary conditions in the normal direction. The structure is illuminated with a normally incident plane wave source, and a reflection monitor was placed above the source.

The first set of simulations are performed without graphene. An $E$-field monitor is placed on the $\mathrm{SiO}_{2} /$ gold interface (where graphene would lie) to compare E-field localizations of different level fractal patterns. Afterward, $1 \mathrm{~nm}$ thick graphene material is added between the $\mathrm{SiO}_{2}$ slab and the gold patterns. Two sets of simulations with different graphene Fermi levels are carried out for each of the first-, second-, and third-level structures. The Fermi level value of the graphene model is taken as $0.2 \mathrm{eV}$ for one set of simulations and $0.7 \mathrm{eV}$ for the other.

Fabrication. To fabricate both of the devices shown in Figure 1, CVD-grown monolayer graphene on $\mathrm{Si} / \mathrm{SiO}_{2}$ samples purchased from Graphene Supermarket is used. For the backgated device, graphene is patterned into $0.5 \mu \mathrm{m}$ by $1 \mu \mathrm{m}$ rectangular active regions by photolithography and $\mathrm{O}_{2}$ plasma etching. Next, source and drain contacts are added by photolithography followed by $400 \mathrm{~nm}$ Au metalization. Finally, to fabricate three different levels of fractals e-beam lithography is done and $5 / 45 \mathrm{~nm} \mathrm{Ti} / \mathrm{Au}$ metal layers are evaporated. After the fabrication steps, the first sample is placed on a printed circuit board (PCB), and copper is used as the back gate. Source and drain contacts are connected onto the PCB by wire bonding.
For the second device, fractal patterns are fabricated on a $\mathrm{Si}$ / $\mathrm{SiO}_{2} /$ graphene sample in the same way as the first device using e-beam lithography. However, in this device a separate $\mathrm{CaF}_{2}$ substrate with a $500 \mathrm{~nm}$ thick gold layer is used for the top gate. For reflection measurements, a $2 \mathrm{~mm}$ by $2 \mathrm{~mm}$ square window opening is obtained using negative photolithography. Subsequently, this top gate is placed with the gold side facing down onto the graphene sample. Double-sided adhesive carbon tapes are used at this stage not only to leave some space between the substrate and the top gate but also as electrical contacts. Finally, this space between graphene and the top gate is filled with ionic liquid, i.e., diethylmethyl(2-methoxyethyl)ammonium, which is commercially available.

\section{ASSOCIATED CONTENT}

\section{Supporting Information}

The Supporting Information is available free of charge on the ACS Publications website at DOI: 10.1021/acsphotonics.6b00440.

Additional details about the fractal metasurfaces, $I_{\mathrm{DS}}-$ $V_{\text {gate }}$ and $I_{\mathrm{DS}}-V_{\mathrm{DS}}$ measurements of the back-gated device, $R-V$ and $C-V$ measurements of the top-gated device, and Raman spectroscopy measurements of graphene (PDF)

\section{AUTHOR INFORMATION}

\section{Corresponding Author}

*E-mail: melis.aygar@bilkent.edu.tr.

\section{Notes}

The authors declare no competing financial interest.

\section{ACKNOWLEDGMENTS}

This work is supported by the projects DPT-HAMIT, NATOSET-193, TUBITAK-113E331, and TUBITAK-114E374. The authors (E.O. and H.C.) also acknowledge partial support from the Turkish Academy of Sciences. One of the authors (H.C.) also acknowledges partial support from a "For Women in Science” fellowship by L'Oréal-Unesco Turkey. H.C. and E.O. contributed equally.

\section{REFERENCES}

(1) Novoselov, K. S. Electric Field Effect in Atomically Thin Carbon Films. Science 2004, 306, 666-669.

(2) Novoselov, K. S.; Geim, A. K.; Morozov, S. V.; Jiang, D.; Katsnelson, M. I.; Grigorieva, I. V.; Dubonos, S. V.; Firsov, A. A. TwoDimensional Gas of Massless Dirac Fermions in Graphene. Nature 2005, 438, 197-200.

(3) Bolotin, K.; Sikes, K.; Jiang, Z.; Klima, M.; Fudenberg, G.; Hone, J.; Kim, P.; Stormer, H. Ultrahigh Electron Mobility in Suspended Graphene. Solid State Commun. 2008, 146, 351-355.

(4) Wang, F.; Zhang, Y.; Tian, C.; Girit, C.; Zettl, A.; Crommie, M.; Shen, Y. R. Gate-Variable Optical Transitions in Graphene. Science 2008, 320, 206-209.

(5) Yao, Y.; Kats, M. A.; Genevet, P.; Yu, N. F.; Song, Y.; Kong, J.; Capasso, F. Broad Electrical Tuning of Graphene-Loaded Plasmonic Antennas. Nano Lett. 2013, 13 (3), 1257-1264.

(6) Yao, Y.; Kats, M. A.; Shankar, R.; Song, Y.; Kong, J.; Loncar, M.; Capasso, F. Wide Wavelength Tuning of Optical Antennas on Graphene with Nanosecond Response Time. Nano Lett. 2014, 14 (1), 214-219.

(7) Dabidian, N.; Kholmanov, I.; Khanikaev, A. B.; Tatar, K.; Trendafilov, S.; Mousavi, S. H.; Magnuson, C.; Ruoff, R. S.; Shvets, G. Electrical Switching of Infrared Light Using Graphene Integration with 
Plasmonic Fano Resonant Metasurfaces. ACS Photonics 2015, 2, 216227.

(8) Echtermeyer, T.; Britnell, L.; Jasnos, P.; Lombardo, A.; Gorbachev, R.; Grigorenko, A.; Geim, A.; Ferrari, A.; Novoselov, K. Strong Plasmonic Enhancement of Photovoltage in Graphene. Nat. Commun. 2011, 2, 458.

(9) Sensale-Rodriguez, B.; Yan, R.; Kelly, M. M.; Fang, T.; Tahy, K.; Hwang, W. S.; Jena, D.; Liu, L.; Xing, H. G. Broadband Graphene Terahertz Modulators Enabled by Intraband Transitions. Nat. Commun. 2012, 3, 780.

(10) Brar, V. W.; Jang, M. S.; Sherrott, M.; Lopez, J. J.; Atwater, H. A. Highly Confined Tunable Mid-Infrared Plasmonics in Graphene Nanoresonators. Nano Lett. 2013, 13, 2541-2547.

(11) Fang, Z.; Wang, Y.; Schlather, A. E.; Liu, Z.; Ajayan, P. M.; Abajo, F. J. G. D.; Nordlander, P.; Zhu, X.; Halas, N. J. Active Tunable Absorption Enhancement with Graphene Nanodisk Arrays. Nano Lett. 2014, 14, 299-304.

(12) Ju, L.; Geng, B.; Horng, J.; Girit, C.; Martin, M.; Hao, Z.; Bechtel, H. A.; Liang, X.; Zettl, A.; Shen, Y. R.; Wang, F. Graphene Plasmonics for Tunable Terahertz Metamaterials. Nat. Nanotechnol. 2011, 6, 630-634.

(13) Falkovsky, L. A.; Pershoguba, S. S. Optical Far-Infrared Properties of a Graphene Monolayer and Multilayer. Phys. Rev. B 2007, 76.

(14) Falkovsky, L. A.; Varlamov, A. A. Space-Time Dispersion of Graphene Conductivity. Eur. Phys. J. B 2007, 56, 281-284.

(15) Adam, S.; Hwang, E. H.; Galitski, V. M.; Sarma, S. D. A SelfConsistent Theory for Graphene Transport. Proc. Natl. Acad. Sci. U. S. A. 2007, 104, 18392-18397.

(16) Li, Z. Q.; Henriksen, E. A.; Jiang, Z.; Hao, Z.; Martin, M. C.; Kim, P.; Stormer, H. L.; Basov, D. N. Dirac Charge Dynamics in Graphene by Infrared Spectroscopy. Nat. Phys. 2008, 4, 532-535.

(17) Polat, E. O.; Kocabas, C. Broadband Optical Modulators Based on Graphene Supercapacitors. Nano Lett. 2013, 13, 5851-5857. 